\title{
Constraints and tests of the OPERA superluminal neutrinos
}

\author{
Xiao-Jun Bi, Peng-Fei Yin, Zhao-Huan Yu, and Qiang Yuan \\ Key Laboratory of Particle Astrophysics, Institute of High Energy Physics, \\ Chinese Academy of Sciences, Beijing 100049, China
}

\begin{abstract}
The superluminal neutrinos detected by OPERA indicates Lorentz invariance violation (LIV) of the neutrino sector at the order of $10^{-5}$. We study the implications of the result in this work. We find that such a large LIV implied by OPERA data will make the neutrino production process $\pi \rightarrow \mu+\nu_{\mu}$ kinematically forbidden for neutrino energy greater than about $5 \mathrm{GeV}$. The OPERA detection of neutrinos at $40 \mathrm{GeV}$ can constrain the LIV parameter to be smaller than $3 \times 10^{-7}$. Furthermore the neutrino decay in the LIV framework will modify the neutrino spectrum greatly. The atmospheric neutrino spectrum measured by IceCube can constrain the LIV parameter to the level of $10^{-12}$. The future detection of astrophysical neutrinos of Galactic sources is expected to be able to give even stronger constraint on the LIV parameter of neutrinos.
\end{abstract}

Introduction - Recently the OPERA collaboration reported an astonishing discovery that the neutrinos from CERN to the Gran Sasso Laboratory may be superluminal [1]. The arrival time of the muon neutrinos with average energy $\sim 17 \mathrm{GeV}$ is earlier by

$$
\delta t=(60.7 \pm 6.9 \text { (stat. }) \pm 7.4 \text { (sys. })) \mathrm{ns}
$$

than that of light, which corresponds to a difference of the muon neutrino velocity and the speed of light of

$\delta_{\nu \gamma}=\left(v_{\nu}-c\right) / c=(2.48 \pm 0.28($ stat. $) \pm 0.30$ (sys. $\left.)\right) \times 10^{-5}$,

with significance level of $6 \sigma$. The energy dependence study of OPERA shows that the velocity difference $\delta_{\nu \gamma}$ is almost independent of energy. It was found that, by splitting the events into two groups with energies below or above $20 \mathrm{GeV}$, the velocity difference is given by $\delta_{\nu \gamma}=(2.16 \pm 0.76 \pm 0.30) \times 10^{-5}$ for $\langle E\rangle=13.9 \mathrm{GeV}$ and $\delta_{\nu \gamma}=(2.74 \pm 0.74 \pm 0.30) \times 10^{-5}$ for $\langle E\rangle=42.9$ $\mathrm{GeV}$. The results above have been confirmed recently by a test performed using a beam with a short-bunch timestructure allowing to measure the neutrino time of flight at the single interaction level. The new analysis show consistent result with that at the first version.

Actually the earlier experiments also gave similar results about the velocity of neutrinos, but with lower significance. In 2007 the MINOS experiment at FERMILAB measured the neutrino speed for energies around 3 $\mathrm{GeV}$ and found [2]

$$
\delta_{\nu \gamma}=(5.1 \pm 2.9) \times 10^{-5},
$$

which seems to be consistent with that by OPERA. Results from even earlier experiments at FERMILAB showed a similar value of $\delta_{\nu \gamma}$ at the order of $10^{-5}[3]$. However, the supernova neutrinos from SN1987A set a much more stringent limit of $\delta_{\nu \gamma}<2 \times 10^{-9}$ for tens of $\mathrm{MeV}$ electron neutrinos [4].

Soon after the OPERA result was reported there were many works to discuss the implications and possible explanations to it [5 -9]. The analysis in these works seem to get a consensus that the effect should be neutrino flavor independent so that the coherent neutrino oscillation is not destroyed. Furthermore, the effect as a function of energy should be a step-like function in order that it is large and energy independent at OPERA and FERMILAB energies $(>\mathrm{GeV})$, while at low energies it is very small to be consistent with the SN1987A result.

Phenomenologically such a superluminal neutrino can be easily accommodated in a Lorentz invariance violation (LIV) model. Coleman and Glashow (hereafter we refer as CG) constructed such a model where LIV was expressed as a modified energy-momentum relation

$$
E^{2}=m^{2}+|\vec{p}|^{2}+\xi|\vec{p}|^{2}
$$

assuming that the Lorentz invariance is violated perturbatively in the context of conventional quantum field theory [10]. In such framework, the neutrino velocity is given by $v_{\nu}=1-m^{2} / 2|\vec{p}|^{2}+\xi / 2$ taking $c=1$. For high energy neutrinos we have $\delta_{\nu \gamma}=\xi / 2$.

In this work we will assume that the LIV term is flavor independent and given by the form in Eq. (4) at high energies. At low energy, the LIV term is suppressed by some specific models, such as that given in 7, [8], to be consistent with SN1987A data. As studied in the CG paper, many phenomenologies changed significantly. Especially for a large $\xi$ of the order $\mathcal{O}\left(10^{-5}\right)$, the processes $\pi^{+} \rightarrow \mu^{+}+\nu_{\mu}$ and $\mu^{-} \rightarrow e^{-}+\nu_{\mu}+\bar{\nu}_{e}$ are even forbidden so that no high energy neutrinos could be produced. In the next section we will show the constraints on the LIV parameters by such processes.

In addition, Eq. (4) can also induce the neutrino radiative decay $\nu_{i} \rightarrow \nu_{j}+\gamma$ and the three body decay $\nu_{i} \rightarrow l_{i}+\nu_{e}+e$ or $\nu_{i} \rightarrow \nu_{i}+e+e$. Such processes will lead to the fast energy loss of neutrinos once the threshold is reached. We will discuss the consequence on the atmospheric and astrophysical neutrino spectra when considering such processes.

Constraints from neutrino related processes - We first consider the process $\pi^{+} \rightarrow \mu^{+}+\nu_{\mu}$. For $\xi>0$, the LIV term in Eq. (4) is equivalent to an "effective" mass term. If this effective mass is too large, we will expect kinematics forbiddance of the pion decay. To derive the threshold condition we get the effective mass of superluminal neu- 
trino following CG [10] as

$$
\left(m_{\nu}^{\text {eff }}\right)^{2} \equiv m_{\nu}^{2}+\xi|\vec{p}|^{2}=m_{\nu}^{2}+2 \delta_{\nu \gamma}|\vec{p}|^{2},
$$

where we take the LIV parameters of pion and muon to be zero as they can be much smaller than the neutrino LIV parameter. We will give a discussion on the LIV parameters of pion and muon in the next section. Neglecting neutrino mass $m_{\nu}$ we get the threshold condition for $\pi^{+}$decay $\pi^{+} \rightarrow \mu^{+}+\nu_{\mu}$ is $m_{\pi} \geq m_{\mu}+m_{\nu}^{\text {eff }} \approx$ $m_{\mu}+E_{\nu} \sqrt{\xi /(1+\xi)}$. In the laboratory frame we have

$$
E_{\nu} \leq\left(m_{\pi}-m_{\mu}\right) \sqrt{1+1 / \xi} .
$$

This inequality means that neutrinos generated by pion decay have a maximum energy beyond which the decay process $\pi^{+} \rightarrow \mu^{+}+\nu_{\mu}$ can not occur at all.

Similarly the processes $K^{+} \rightarrow \mu^{+}+\nu_{\mu}, \mu \rightarrow \nu_{\mu}+e+\bar{\nu}_{e}$ and $\tau \rightarrow \nu_{\tau}+e(\mu)+\bar{\nu}_{e(\mu)}$ should also be forbidden at high energies. In Fig. 1 we show the constraints on the neutrino velocity $\left(v_{\nu}-c\right) / c$ for different neutrino energies with different processes. The region above the line should be excluded if we detect the neutrinos with corresponding energies. The experimental data by FERMILAB79, MINOS and OPERA are also shown. From Fig. 1 we can see that the process $\pi \rightarrow \mu \nu_{\mu}$, through which the OPERA neutrinos are produced, gives the most stringent constraint. Process $\mu \rightarrow \nu_{\mu}+e+\bar{\nu}_{e}$ gives a similar but a little weaker constraint. It is shown that the detection of neutrinos with energies as high as $43 \mathrm{GeV}$ by OPERA can set a constraint $\delta_{\nu \gamma}<3 \times 10^{-7}$, while the $\sim 200 \mathrm{GeV}$ neutrinos detected at Fermilab give $\delta_{\nu \gamma}<2 \times 10^{-8}$.

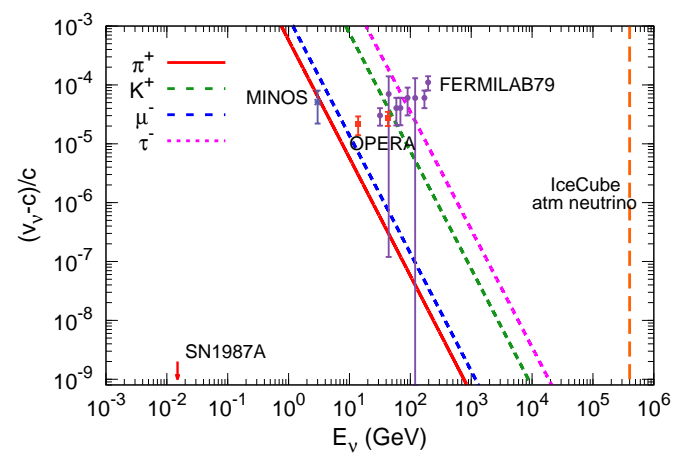

FIG. 1: The neutrino velocity constrained by the neutrino energy, for processes $\pi(K)^{+} \rightarrow \mu^{+} \nu_{\mu}, \mu(\tau) \rightarrow \nu_{\mu(\tau)}+e(\mu)+$ $\bar{\nu}_{e(\mu)}$.

Actually the highest energy neutrinos detected are the atmospheric neutrinos at IceCube, which reach $\sim 400$ $\mathrm{TeV}$ [1]. These neutrinos are generated by the same process as OPERA neutrinos, i.e. through $p p \rightarrow \pi(K) \rightarrow$ $\mu+\nu_{\mu}$. From Fig. 1 we can see that the IceCube neutrinos can constrain LIV parameter down to $\sim 4 \times 10^{-15}$.

In the following we extend the CG model with a more general case that the dispersion relation is modified as

$$
E^{2}=m^{2}+|\vec{p}|^{2}+\beta|\vec{p}|^{\alpha} .
$$

We use the same particle decay processes to constrain the model parameters. The constraints from neutrino energy for $\pi^{+} \rightarrow \mu^{+}+\nu_{\mu}$ are shown in Fig. 2], for different values of $\alpha$. The results show that larger $\alpha$ will give weaker constraints on the parameters.

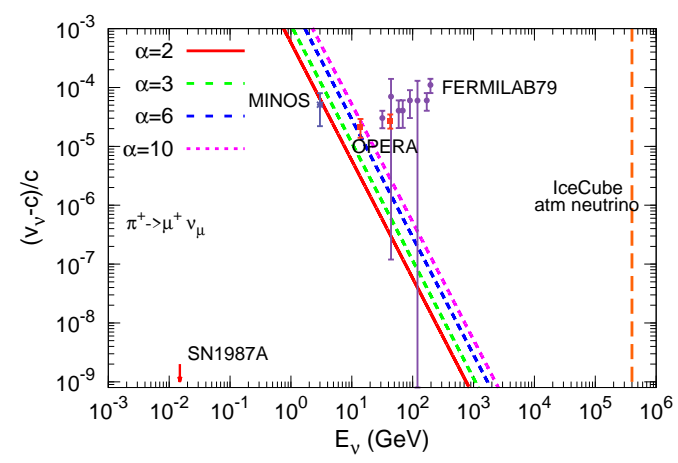

FIG. 2: The neutrino velocity constrained by the neutrino energy for the process $\pi^{+} \rightarrow \mu^{+} \nu_{\mu}$, for different values of $\alpha$.

The atmospheric and astrophysical neutrino spectraWe have discussed the constraints on the LIV parameter of neutrinos according to the processes of $\pi \rightarrow \mu+\nu_{\mu}$ and $\mu \rightarrow \nu_{\mu}+e+\nu_{e}$. These processes can only constrain the difference of the LIV parameters between the parent particle and the daughter particles. Therefore to give stronger constraint on the neutrino LIV parameter we have to give constraints on $\pi$ and $\mu$ first.

For superluminal $\pi^{+}$it will lose energy quickly by radiative emission $\pi^{+} \rightarrow \pi^{+} \gamma$. IceCube experiment has measured the atmospheric neutrino spectrum up to $\sim 400 \mathrm{TeV}$, which fits very well with the model calculation [11]. This means that the high energy charged pions do not lose much energy before they decay to neutrinos. This can give a strong constraint on the process $\pi^{+} \rightarrow \pi^{+} \gamma$. The threshold of this process is $E_{\pi}>m_{\pi} / \sqrt{c_{\pi}^{2}-c^{2}}=m_{\pi} / \sqrt{\xi_{\pi}}[10]$. This leads to an upper bound of $\xi_{\pi}<10^{-14}$ when the maximum energy of pion is about $2 \mathrm{PeV}$.

If the maximum attainable velocity of muon is smaller than $c$, it is still possible to have the process $\pi \rightarrow \mu+\nu_{\mu}$ with superluminal $\nu_{\mu}$. In such a case the LIV parameter is constrained by the process $\gamma \rightarrow \mu \mu$. As the $\gamma$ energy as high as $80 \mathrm{TeV}$ has been detected at Crab Nebula [12], we have $\left|\xi_{\mu}\right|<10^{-11}$. Therefore the LIV parameter of neutrinos can be constrained at most to the order $\sim 10^{-11}$ according to pion decay 13. More stringent constraint on the flavor dependent LIV parameter was set in [14].

In the following we study how to give more stringent constraints on the neutrino LIV parameter. For superluminal neutrino with the LIV dispersion relation Eq. (4), it will lose energy through radiative decay $\nu_{i} \rightarrow \nu_{j}+\gamma$ or the three body decay. The three body decay can be a charged current (CC) process $\nu_{\mu} \rightarrow \mu+\nu_{e}+e$ or a neutral current (NC) process $\nu_{\mu} \rightarrow \nu_{\mu}+e+e$. Among these 
three processes, the $\mathrm{CC}$ process has the largest decay rate, but the threshold energy is also the highest. The radiative process has the lowest decay rate with lowest threshold energy. In [9] the OPERA neutrino energy loss is studied when propagating from CERN to Gran Sasso taking $\xi=5 \times 10^{-5}$. As we have shown that neutrinos with energy up to tens of $\mathrm{GeV}$ can not be produced if $\xi$ is so large, we will study the energy loss process with a much smaller $\xi$. As argued above if muons are involved, the LIV parameter of neutrinos can only be constrained to the order $10^{-11}$. Therefore in the following we focus on the NC process which involves electrons in the final states, as discussed in [9]. The processes $e \rightarrow e \gamma$ and $\gamma \rightarrow$ ee give $\left|\xi_{e}\right|<10^{-15}$ by observation of the Crab Nebula [12].

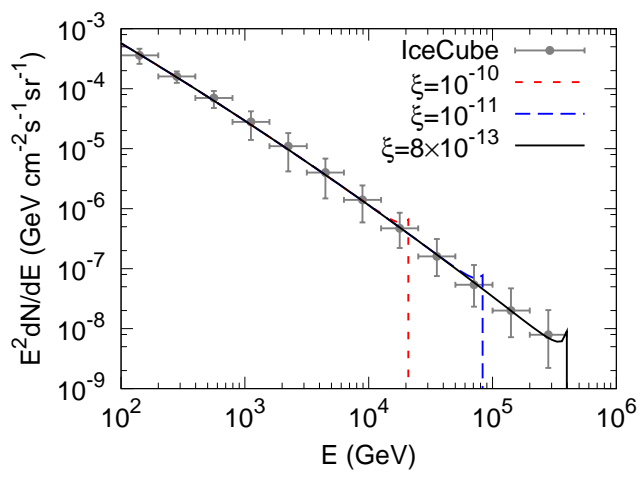

FIG. 3: The expected atmospheric neutrino spectra with LIV parameters $\xi=10^{-10}, 10^{-11}$ and $8 \times 10^{-13}$ respectively. The measured data by IceCube is also plotted [11].

The calculation of the three body decay rate is very complicated if the LIV effect of final state particles is taken into account. Working out the integration of final state phase space, we get

$$
\frac{\mathrm{d} E}{\mathrm{~d} t}=-\langle\Delta E\rangle \Gamma \approx-10^{-3}\left(\frac{E}{\mathrm{TeV}}\right)^{6}\left(\frac{\xi}{10^{-10}}\right)^{3} \mathrm{GeV} \mathrm{s}^{-1}
$$

where $\langle\Delta E\rangle$ and $\Gamma$ are the averaged energy loss of each decay and the decay width. A detailed calculation in the laboratory system has been given in [15].

To calculate the neutrino spectra with the energy loss due to decay, we follow the way of calculating the spectra of ultra high energy (UHE) protons propagating in the cosmic microwave background (CMB) field [16]. The detected neutrino spectra can be written as

$$
\phi(E)=\frac{q_{0}\left(E_{0}\right)}{4 \pi d^{2}} \frac{\mathrm{d} E_{0}}{\mathrm{~d} E}
$$

where $q_{0}\left(E_{0}\right)$ is the production spectra per unit time at the source, $d$ is the distance of the source, $E_{0}$ and $E$ are the energies at the source and detector respectively. The energy interval dilation $\mathrm{d} E_{0} / \mathrm{d} E$ is

$$
\frac{\mathrm{d} E_{0}}{\mathrm{~d} E}=\exp \left[\int_{0}^{t=d / c} \frac{\mathrm{d} b\left[E_{0}\left(t^{\prime}\right)\right]}{\mathrm{d} E_{0}\left(t^{\prime}\right)} \mathrm{d} t^{\prime}\right]
$$

where $b(E) \equiv-\mathrm{d} E / \mathrm{d} t$ is the energy loss rate in Eq. (8). Note here we consider the local neutrino sources. For cosmological sources as discussed in [16] Eqs. (9) and (10) are more complicated.

We first study the atmospheric neutrinos. In [11] the up-going neutrinos from $100 \mathrm{GeV}$ to $400 \mathrm{TeV}$ were detected by IceCube. We take a rough travel distance of $10^{4} \mathrm{~km}$ for the neutrinos with zenith angle $124^{\circ}-180^{\circ}$. In Fig. 3. we show the neutrino spectra with LIV parameters $\xi=10^{-10}, 10^{-11}$, and $8 \times 10^{-13}$ respectively. It is shown that for specified distance of the source, there is a maximum energy of the neutrinos above which the energy loss is too fast that the neutrinos can not reach us with high energies. According to the energy loss rate in Eq. (8), we have $E_{\mathrm{GeV}}=\left(E_{0, \mathrm{GeV}}^{-5}+5 \times 10^{9} \xi^{3} t_{\mathrm{sec}}\right)^{-1 / 5}$. For $E_{0} \rightarrow \infty$, $E_{\max } \propto t^{-1 / 5}=(d / c)^{-1 / 5}$. For $\xi=8 \times 10^{-13}$ we have $E_{\max } \approx 400 \mathrm{TeV}$ for atmospheric neutrinos. According to the fact that IceCube has detected atmospheric neutrinos up to $400 \mathrm{TeV}$, we can set a bound of the LIV parameter of neutrino $\xi \lesssim 8 \times 10^{-13}$.

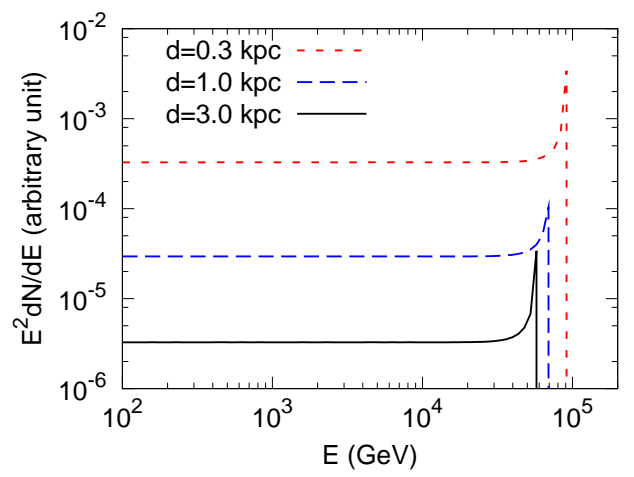

FIG. 4: Expected neutrino spectra with LIV parameter $\xi=$ $10^{-15}$, for sources at distances $0.3,1.0$ and $3.0 \mathrm{kpc}$ respectively. The source spectrum is assumed as $E^{-2}$.

Then we study the effect of energy loss on the astrophysical neutrino spectrum. Because these neutrinos will propagate very long distance before reaching the Earth we expect the spectral measurement can probe even smaller LIV parameters of neutrinos. Up to now, no high energy astrophysical neutrinos were detected. However, as the well believed sources of Galactic cosmic rays (CRs), supernova remnants (SNRs) are expected to be good candidates of astrophysical neutrino sources. Fig. 4 gives an illustration of the astrophysical neutrino spectra taking into account the LIV effect with much smaller parameter $\xi=10^{-15}$. Three distances of the sources, $0.3,1.0$ and $3.0 \mathrm{kpc}$ are adopted. In the calculation the source spectrum is assumed to be $\propto E^{-2}$. We can see 
that for sources with distance $\sim \mathrm{kpc}$ the cutoff energy is about several tens to $100 \mathrm{TeV}$ for this small LIV parameter. The $\gamma$-ray observations of the Galactic SNRs show that the accelerated energies of particles can well exceed $\mathrm{TeV}$, which enables the production of neutrinos with energies much higher than $\mathrm{TeV}$. Therefore the observation of cutoff at neutrino spectra in the future can set a very strong constraints on the LIV parameter.

Finally the Greisen-Zatsepin-Kuzmin (GZK) neutrinos are expected when the ultra-high energy cosmic rays (UHECRs) interact with the CMB photons and generate the GZK cutoff of UHECRs [17]. It is expected that the IceCube might discover such neutrinos in the near future. However, as shown above the decay mean free path for tens $\mathrm{TeV}$ neutrinos is only of $\mathrm{kpc}$ scale. We might have no chance to detect any neutrinos from extragalactic sources if they are superluminal. LIV at the proton sector may also suppress the GZK neutrinos [18].

Conclusion -We studied the implications of the claim of superluminal neutrinos by the OPERA collaboration. The observation generally indicates LIV in the neutrino sector. In such a framework, we find the neutrino production process $\pi^{+} \rightarrow \mu^{+} \nu_{\mu}$ becomes kinematically forbidden for $E_{\nu}>5 \mathrm{GeV}$ if taking $\delta_{\nu \gamma}=2.5 \times 10^{-5}$ as shown by OPERA data. The detection of $\sim 40 \mathrm{GeV}$ and $\sim 200 \mathrm{GeV}$ neutrinos by OPERA and Fermilab can constrain the LIV parameters to $3 \times 10^{-7}$ and $2 \times 10^{-8}$ respectively.
Then we discuss if it is possible to constrain the neutrino LIV parameters by studying the three body decay process of neutrinos. Such process will modify the atmospheric and astrophysical neutrino spectra. Because there is no spectral cutoff of the atmospheric neutrinos up to $\sim 400 \mathrm{TeV}$ as measured by IceCube [11], the neutrino LIV parameter can be constrained to less than $10^{-12}$.

We further studied the astrophysical neutrino spectra from distant sources, for example the Galactic SNRs, which are usually taken as the sources of Galactic cosmic rays. The long travel distance makes it possible to probe even smaller LIV parameters. As an illustration, we find that for $\xi \sim 10^{-15}$ and source distance $\sim \mathrm{kpc}$, the decay process is expected to result in a distinctive spectral cutoff of the neutrino spectra at energies about several tens $\mathrm{TeV}$. This effect can be tested through the future detection of the neutrino sources.

\section{Acknowledgments}

We thank Prof. X. M. Zhang and Prof. H. B. Hu for helpful discussions. This work is supported by the Natural Science Foundation of China under the grant NO. 11075169, and the 973 project under grant No. 2010CB833000 and by the Chinese Academy of Science under Grant No. KJCX2-EW-W01.
[1] T. Adam et al. [OPERA Collaboration], arXiv: 1109.4897.

[2] P. Adamson at al. [MINOS Collaboration], Phys. Rev. D 76 (2007) 072005.

[3] G. R. Kalbfleisch, N. Baggett, E. C. Fowler, J. Alspector, Phys. Rev. Lett. 43, 1361 (1979); J. Alspector et al., Phys. Rev. Lett. 36, 837 (1976).

[4] M. J. Longo, Phys. Rev. D 36, 3276 (1987); K. Hirata et al., Phys. Rev. Lett. 58, 1490 (1987); R. M. Bionta et al., Phys. Rev. Lett. 58, 1494 (1987).

[5] For some earlier works on superluminal neutrinos, see J. Alfaro, Phys. Rev. Lett. 94, 221302 (2005). hep-th/0412295; H. Pas, S. Pakvasa, T. J. Weiler, Phys. Rev. D72, 095017 (2005). hep-ph/0504096; S. Hollenberg, O. Micu, H. Pas, T. J. Weiler, Phys. Rev. D80, 093005 (2009). arXiv:0906.0150 [hep-ph]].

[6] G. Cacciapaglia, A. Deandrea and L. Panizzi, arXiv:1109.4980 [hep-ph]; G. Amelino-Camelia, G. Gubitosi, N. Loret, F. Mercati, G. Rosati and P. Lipari, arXiv:1109.5172 [hep-ph]; D. Autiero, P. Migliozzi and A. Russo, arXiv:1109.5378 [hep-ph]; J. Ciborowski and J. Rembielinski, arXiv:1109.5599 [hep-ex]; J. Alexandre, arXiv:1109.5629 [hep-ph]; F. R. Klinkhamer, arXiv:1109.5671 [hep-ph]; S. S. Gubser, arXiv:1109.5687 [hep-th]; R. B. Mann, U. Sarkar, arXiv:1109.5749 [hep-ph]]; M. Li and T. Wang, arXiv:1109.5924 [hepph]; C. Pfeifer, M. N. R. Wohlfarth, arXiv:1109.6005 [gr-qc]]; Z. Lingli and B. Q. Ma, arXiv:1109.6097 [hep-ph]; R. A. Konoplya, arXiv:1109.6215 [hep-th]];
L. Iorio, arXiv:1109.6249 [gr-qc]]; J. Alexandre, J. Ellis and N. E. Mavromatos, arXiv:1109.6296 [hep-ph]; M. A. Anacleto, F. A. Brito, E. Passos, arXiv:1109.6298 [hep-th]]; S. Gardner, arXiv:1109.6520 [hep-ph]]; F. R. Klinkhamer, G. E. Volovik, arXiv:1109.6624 [hepph]]; M. Matone, arXiv:1109.6631 [hep-ph]]; E. Ciuffoli, J. Evslin, J. Liu, X. Zhang, arXiv:1109.6641 [hep-ph]]; P. Wang, H. Wu and H. Yang, arXiv:1109.6930 [hep-ph]. R. Cowsik, S. Nussinov and U. Sarkar, arXiv:1110.0241 [hep-ph]; R. Torrealba, arXiv:1110.0243 [hep-ph]; P. Wang, H. Wu and H. Yang, arXiv:1110.0449 [hep-ph]. E. N. Saridakis, arXiv:1110.0697 [gr-qc].

[7] G. Dvali and A. Vikman, arXiv:1109.5685 [hep-ph].

[8] G. F. Giudice, S. Sibiryakov and A. Strumia, arXiv:1109.5682 [hep-ph].

[9] A. G. Cohen, S. L. Glashow, arXiv:1109.6562 [hep-ph]].

[10] S. R. Coleman and S. L. Glashow, Phys. Rev. D 59, 116008 (1999) arXiv:hep-ph/9812418.

[11] R. Abbasi et al. [IceCube Collaboration], Phys. Rev. D83:012001 (2011).

[12] F. Aharonian et al. [ The HEGRA Collaboration ], Astrophys. J. 614, 897-913 (2004). astro-ph/0407118.

[13] A. Kostelecky, N. Russell, Rev. Mod. Phys. 83:11 (2011).

[14] R. Cowsik and B. V. Sreekantan, Phys. Lett. B 449, 219 (1999) hep-ph/9811241.

[15] S. Mohanty and S. Rao, arXiv:1111.2725 [hep-ph]; M. Li, D. Liu, J. Meng, T. Wang and L. Zhou, arXiv:1111.3294 [hep-ph]. Y. Huo, T. Li, Y. Liao, D. V. Nanopoulos and Y. Qi, arXiv:1112.0264 [hep-ph]. 
[16] V. Berezinsky, A. Z. Gazizov, S. I. Grigorieva, Phys. Rev. D74, 043005 (2006). hep-ph/0204357.

[17] S. Yoshida, M. Teshima, Prog. Theor. Phys. 89, 833-845 (1993); O. E. Kalashev, V. A. Kuzmin, D. V. Semikoz, G. Sigl, Phys. Rev. D66, 063004 (2002); R. Engel,
D. Seckel, T. Stanev, Phys. Rev. D64, 093010 (2001).

[18] S. T. Scully, F. W. Stecker, Astropart. Phys. 34, 575-580 (2011). 